\title{
Ökonomisierung als Chance für das Gesundheitswesen
}

\section{Economization as an Opportunity for the Health Care System}

\author{
Gregor Zünd \\ Spitaldirektion, Universitätsspital Zürich
}

Ökonomisierung in der Medizin ist ein Schlagwort. Je nachdem, in welchem Kontext von Ökonomisierung die Rede ist, sind verschiedene Beweggründe damit verbunden: Die einen verbinden damit das Gefühl, dass die Gesundheitsversorgung mehr und mehr durch Krankenhausmanager geführt wird statt durch Mediziner und Pflegende. Dahinter verbirgt sich ein Gefühl des Machtverlustes der Medizinalberufe.

Andere sehen in der Ökonomisierung einen Prozess, bei dem mehr und mehr private Anbieter die Gesundheitsversorgung übernehmen beziehungsweise öffentliche Einrichtungen privatisiert werden. Dahinter steckt ein vermuteter Machtverlust der öffentlichen Hand. Wiederum andere sehen in der Ökonomisierung die zunehmende Tendenz, einseitig auf die Kosten im Gesundheitswesen zu blicken. Dahinter liegt die Befürchtung, dass das Gesundheitssystem eines Tages nicht mehr bezahlbar ist.

\section{Der Kostenröhrenblick und seine Folgen}

Tatsächlich wurde in den vergangenen Jahren das Gesundheitssystem in der Öffentlichkeit zunehmend aus der Kostenperspektive thematisiert. Die steigenden Gesundheitskosten und damit die steigenden Krankenkassenprämien haben in den Medien wie auch in der Politik zu einem sogenannten «Kostenröhrenblick» geführt. So sind die Ankündigungen der Prämien des kommenden Jahres im Herbst alljährlich Anlass für gesundheitspolitische Stellungnahmen der Akteure des Gesundheitssystems.

Dieser eingeschränkte Blick bleibt nicht ohne Wirkung in der Schweizer Bevölkerung: Das Credit-Suisse-Sorgenbarometer zeigt auf, dass die Gesundheit und die Krankenkassen in den Augen der Bevölkerung 2018 die zweitgrössten Probleme des Landes darstellen. Insgesamt 41 Prozent der Schweizerinnen und Schweizer sahen darin einen Problembereich, der ihnen die meisten Sorgen bereitet.

Darauf reagierten auch die politischen Parteien: So hat die CVP Schweiz (Christlichdemokratische Volkspartei) die Kostenbremsen-Initiative vorgelegt: Steigen die Kosten jährlich stärker als die Löhne, muss der Bund zusammen mit Kantonen, Krankenversicherern und den Leistungserbringern zwingend Kostensenkungsmassnahmen durchsetzen. Die SP wiederum will mit der Prämien-Entlastungs-Initiative, dass die Haushalte nicht mehr als 10\% ihres Einkommens für die Prämien aufwenden müssen. Auch Bund und Kantone setzen vermehrt bei den Kosten an: Der Bundesrat hat 2018 ein Kostensenkungsprogramm in der Grundversicherung mit zwölf Massnahmen verabschiedet, die nun nach und nach umgesetzt werden sollen. Eine Reihe von Kantonen versuchen die Mengen bei Krankenhäusern zu steuern, indem sie die Zahl der Krankenhäuser festlegen, welche vom Kanton einen Leistungsauftrag erhalten, sowie den Krankenhäusern den Umfang des Leistungsauftrags vorschreiben.

Solche Massnahmen sind populär, weil sie eine unmittelbare Wirkung versprechen. Die Erfahrungen der letzten Jahrzehnte haben uns aber gelehrt, dass der gewünschte Effekt meist nicht eintritt. Das Gesundheitssystem ist zu komplex, als dass sich durch das Umlegen einzelner Schalthebel massgebliche Veränderungen erreichen lassen. So hat die Einführung von Mindestfallzahlen einen Anreiz geschaffen, dass Krankenhäuser mit ungenügenden Fallzahlen unnötige OPs durchführen, um die Kriterien zu erfüllen.

\section{Ökonomie und Medizin als Zwillingsschwestern}

Angesichts weiterhin steigender Gesundheitskosten werden wir uns auch in den kommenden Jahren mit der Kostenfrage und der Ökonomisierung der Medizin auseinandersetzen müssen. Und das zu Recht: Gesundheitsversorgung hat immer mit Ökonomie zu tun, aber nicht nur mit Kosten. Wenn wir Ärztemangel erleben oder unter Zeitdruck stehen, dann haben wir eine klassische ökonomische Problemstellung: Die Knappheit an Ressourcen löst eine Suche nach effizienterer Produktion aus. Oder nach gezielterer Indikationsstellung.

In diesem Sinne steht fest, dass Ökonomie die Zwillingsschwester der Medizin ist, wenn es um die Gesund- 
heitsversorgung und das Gesundheitssystem geht. Die beiden gehen Hand in Hand. Das bringt auch das KVG zum Ausdruck, wenn es vorschreibt, dass alle Leistungen, die von der obligatorischen Krankenpflegeversicherung übernommenen werden, wirksam, zweckmässig und wirtschaftlich sein müssen.

Es erstaunt daher auch nicht, wenn eine der grössten Neuerungen in der Betrachtung der Gesundheitssysteme der letzten Jahrzehnte von Ökonomen stammt, und nicht von Medizinern oder Gesundheitspolitikern. Michael E. Porter und Elisabeth Olmsted Teisberg von der Harvard Business School haben 2006 das wohl einflussreichste Buch der nationalen Gesundheitssysteme der jüngeren Vergangenheit geschrieben: «Redefining Healthcare Creating Value-Based Competition on Results» [1].

Die beiden Autoren setzten den Patientennutzen ins Zentrum und schlugen vor, die Akteure möchten sich künftig in einem Wettbewerb um diesen Nutzen messen und nicht mehr um die Menge der Gesundheitsleistungen. Dies wird auch mit dem Schlagwort «From volume to value» bezeichnet. Gemäss diesem Konzept wird Nutzen definiert als Qualität im Verhältnis zu den Kosten, die dafür notwendig waren (Abb. 1). Mit anderen Worten: Der Patient/die Patientin und die Gesellschaft sollen das Bestmögliche zum attraktivsten Preis erhalten.

\section{Investitionen in Qualität müssen sich lohnen}

Von diesem Ziel, eines werteorientierten Gesundheitssystems, sind wir allerdings noch weit entfernt. Tatsächlich wissen wir mit unserem Kostenröhrenblick sehr viel über die direkten Kosten, die etwa eine Behandlung verursacht: Jeden Verband, jede medizinische oder pflegerische Handlung können wir quantifizieren und abrechnen. Hingegen wissen wir über die Folgen unserer Leistungen noch viel zu wenig. Es ist an der Zeit, dass wir die Wirkung unseres Tuns auf die Patientengesundheit besser messen.

Es reicht in Zukunft nicht mehr, wenn wir alleine die Patientenzufriedenheit, die Mortalität und Infektionsraten erfassen. Die Krankenhäuser sollten den gesundheitlichen Erfolg ihres Wirkens viel genauer überprüfen und untereinander vergleichen. Das International Consortium for Health Outcomes Measures (ICHOM) hat in der Folge der Initiative von Porter und Olmsted Teisberg bisher 28 Standardsets zu verschiedenen Indikationen erarbeitet, vom Vorhofflimmern bis zur Makuladegeneration. Die aufgearbeiteten Krankheiten decken rund $60 \%$ der weltweiten Krankheitslast ab. Die ICHOM-Standardsets enthalten multidisziplinär erarbeitete Outcome-Indikatoren, die sich am Patientennutzen orientieren und den Weg weisen zu einer umfassenden Wirkungsprüfung und zu einem Vergleich zwischen verschiedenen Leistungserbringern.

Outcomes an einem Krankenhaus zu etablieren und zu erfassen, ist ein langer, steiniger und teurer Weg. Aber er

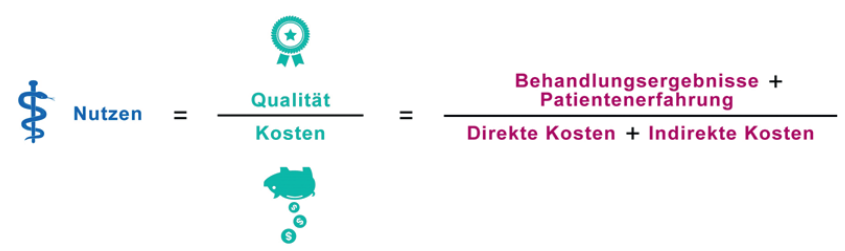

Abbildung 1. Nutzen bringt Kosten und Qualität ins Verhältnis.

lohnt sich: Er führt zu mehr Transparenz, besserem Einbezug der Patienten, mehr Qualität und letztlich auch zu mehr Nutzen. Allerdings werden diese Bemühungen im jetzigen regulatorischen Umfeld nicht honoriert. Investitionen in die Qualität haben sich bisher kaum ausbezahlt. Mit der neu gültigen Verordnung über die Integrität und Transparenz im Heilmittelbereich (VITH) macht der Bund aber einen Schritt in die richtige Richtung: Neu können Versicherer und Leistungserbringer vereinbaren, dass Rabatte aus dem Einkauf von Medikamenten nicht vollumfänglich an die Versicherer weitergegeben werden. Sie können Einkünfte aus Rabatten dann einbehalten, wenn sie nachweislich zur Verbesserung der Qualität der Behandlung eingesetzt werden. Es bleibt zu hoffen, dass diese Regelung nicht die einzige bleibt, die Nutzenorientierung fördert.

\section{Fazit}

Statt über die «Ökonomisierung der Medizin» zu klagen oder diese passiv hinzunehmen, kann die gegenwärtige Situation genutzt werden, um sich der Ziele im Gesundheitswesen zu vergewissern und Strukturen und Prozesse auf die gewünschten Ergebnisse auszurichten. Dies hat die Schweizerische Akademie der Medizinischen Wissenschaften in einem Positionspapier zu diesem Thema vor fünf Jahren festgehalten. Dieser Aussage gibt es nichts hinzuzufügen. Ökonomische Ansätze und Modelle wie die «Value-Based Healthcare» können wertvolle Impulse geben, damit wir die Gesundheitsversorgung transparenter, effizienter und patientenzentriert weiterentwickeln. Und dadurch mehr Nutzen für die Patienten und die Gesellschaft erzeugen.

\section{Bibliografie}

1. Porter ME, Olmsted Teisberg E: Redefining Healthcare - Creating Value-Based Competition on Results. Brighton, MA; Harvard Business School Press: 2006.

\section{Prof. Dr. Gregor Zünd}

CEO/Vorsitzender der Spitaldirektion

Universitätsspital Zürich

Rämistrasse 100

8091 Zürich

gregor.zuend@usz.ch 